\title{
The Relation Reflection Scheme
}

\author{
Peter Aczel \\ petera@cs.man.ac.uk \\ Schools of Mathematics and Computer Science \\ The University of Manchester
}

September 14, 2007

\section{Introduction}

In this paper we introduce a new axiom scheme, the Relation Reflection Scheme (RRS), for constructive set theory. Constructive set theory is an extensional set theoretical setting for constructive mathematics. A formal system for constructive set theory was first introduced by Myhill in [8]. In $[1,2,3]$ I introduced a formal system CZF that is closely related to Myhill's formal system and gave a natural interpretation of $\mathbf{C Z F}$ and extensions of it in Martin-Löf's constructive type theory, [7]. The axiom system $\mathbf{C Z F}$ can be formulated in the same first order language as that of $\mathbf{Z F}$, but uses intuitionistic logic rather than classical logic. But when the law of excluded middle is added the resulting classical theory has the same theorems as ZF. So, from the classical point of view, CZF does not involve any choice principle. The axiom $\mathbf{C C}$ of countable choice and even the stronger axiom $\mathbf{D C}$ of dependent choices have been accepted principles of constructive mathematics that have played an important role in the development of constructive analysis, particularly Bishop style constructive mathematics. In fact the interpretation of CZF in constructive type theory actually also gives an interpretation of each instance of the axiom scheme RDC of relative dependent choices; an axiom scheme that implies DC. So it is natural to consider allowing the free use of RDC in constructive set theory. But there are reasons for avoiding the use of any choice principles, even of $\mathbf{C C}$, when possible. The Boolean-valued models of $\mathbf{Z F}$ generalise to Heyting-valued models of CZF. More precisely, in CZF, given a set-presented frame, which will form a complete Heyting algebra, $\Omega$, there is a natural $\Omega$-valued model construction that gives a reinterpretation of $\mathbf{C Z F}$, [5, 6], and, in general, these reinterpretations do not 
always model any choice principles, even the countable choice principle. For example, countable choice fails in the Heyting valued model over the complete Heyting algebra of open sets of real numbers; see Proposition 2.2 of chapter 15 in volume II of [9]. So, if one wants to prove results that will hold in all such Heyting-valued models one should try to avoid the use of any choice principles in constructive set theory, when possible.

One of the axiom schemes of $\mathbf{C Z F}$ is the subset collection scheme. In this paper that scheme does not play a role so that we prefer to state our results for the axiom system $\mathbf{C Z F}^{-}$obtained from $\mathbf{C Z F}$ by leaving out the subset collection scheme.

The axiom scheme RRS can be derived in $\mathbf{C Z F}^{-}+\mathbf{R D C}$. In fact, in $\mathbf{C Z F}^{-}$the scheme RDC can be analysed as the combination of $\mathbf{R R S}$ with DC. While RDC and DC are clearly choice principles I claim that $\mathbf{R R S}$ is not a choice principle. Moreover, assuming $\mathbf{C Z F}^{-}+\mathbf{R R S}$ as metatheory, all Heyting-valued models using set-generated frames do reinterpret $\mathbf{C Z F}^{-}+$ $\mathbf{R R S}$, a property of $\mathbf{C Z F}^{-}+\mathbf{R R S}$ that is not shared with $\mathbf{C Z F} \mathbf{F}^{-}+\mathbf{R D C}$. It is plausible, but I have not seen a proof, that there are instances of RDC that are not theorems of $\mathbf{C Z F}+\mathbf{D C}$, and in that case RRS would also not be a theorem of $\mathbf{C Z F}+\mathbf{D C}$ even though it is a theorem of $\mathbf{Z F}$, as we will show.

We will show that $\mathbf{R R S}$ is useful by proving a result, in $\mathbf{C Z F}^{-}+\mathbf{R R S}$, about coinductive definitions of classes that is a dual to a basic result about inductive definitions of classes. An earlier proof of the coinduction result used RDC and it was as a consequence of my dissappointment with the need to use a choice principle that I came to consider RRS. Coinductive definitions promiss to be useful in constructive mathematics. For example, when inductively generating a cover relation in formal topology it is natural to also coinductively generate a binary positivity relation.

We introduce the new scheme, RRS, in Section 2 after giving the standard formulations of $\mathbf{D C}$ and $\mathbf{R D C}$ and show that in $\mathbf{C Z F}{ }^{-}$the scheme RDC can be analysed as RRS + DC. We also show that RRS is a theorem of $\mathbf{Z F}$. In Section 3 we prove the result about coinductive definitions of classes in $\mathbf{C Z F}^{-}+\mathbf{R R S}$. In Section 4 we review the Heyting-valued model construction for $\mathbf{C Z F}^{-}$and in Section 5 we show that each instance of RRS holds in such a model.

We recall that the axiom system $\mathbf{C Z F}{ }^{-}$may be axiomatised in the same first order language as the axiom system for classical axiomatic set theory, but uses intuitionistic logic, the standard axioms of Extensionality, Pairing, Union and Infinity and the axiom schemes of Restricted Separation, Set Induction and Strong Collection. The reader should refer to [4] for more information concerning constructive set theory. We will use standard class 
notation and terminology as in [4]. For example we may state the Strong Collection Scheme, used several times in this paper, in the following informal way.

Definition: 1.1 (Strong Collection Scheme) Given a class $R$ of ordered pairs, for every set a such that

$$
(\forall x \in a) \exists y(x, y) \in R
$$

there is a set $b$ such that $(\forall x \in a)(\exists y \in b)(x, y) \in R \quad$ and $\quad(\forall y \in b)(\exists x \in$ a) $(x, y) \in R$.

\section{The new scheme}

We recall the axiom $\mathbf{D C}$ and the scheme RDC:

Definition: 2.1 (Axiom of Dependent Choices, DC) For sets $A, R$ with $R \subseteq A \times A$, such that

$$
(\forall x \in A)(\exists y \in A)(x, y) \in R
$$

if $a_{0} \in A$ then there is $f: \mathbb{N} \rightarrow A$ such that $f(0)=a_{0}$ and for all $n \in \mathbb{N}$, $(f(n), f(n+1)) \in R$.

Definition: 2.2 (Scheme of Relative Dependent Choices, RDC) For classes $A, R$ with $R \subseteq A \times A$, such that

$$
(\forall x \in A)(\exists y \in A)(x, y) \in R
$$

if $a_{0} \in A$ then there is $f: \mathbb{N} \rightarrow A$ such that $f(0)=a_{0}$ and, for all $n \in \mathbb{N}$, $(f(n), f(n+1)) \in R$.

Next we formulate the new scheme.

Definition: 2.3 (Relation Reflection Scheme, RRS) For classes $A, R$ with $R \subseteq A \times A$, such that

$$
(\forall x \in A)(\exists y \in A)(x, y) \in R
$$

if $a$ is a subset of $A$ then there is a subset $b$ of $A$ such that $a \subseteq b$ and $(\forall x \in b)(\exists y \in b)(x, y) \in R$.

This scheme is a consequence of RDC. In fact we have the following result. 
Theorem: $2.4\left(\mathbf{C Z F}^{-}\right)$The scheme $\mathbf{R D C}$ is equivalent to $\mathbf{R R S}+\mathbf{D C}$.

Proof: Clearly DC can be proved using $\mathbf{R D C}$ as each set is a class. Next we prove each instance of RRS using RDC. So let $A, R$ be classes such that

$$
(\forall x \in A)(\exists y \in A)(x, y) \in R
$$

and let $a$ be a subset of $A$. Let $A^{\prime}=\operatorname{Pow}(A)$, the class of all subsets of $A$ and let $R^{\prime}$ be the class of all pairs $(X, Y) \in A^{\prime} \times A^{\prime}$ such that

$$
(\forall x \in X)(\exists y \in Y)(x, y) \in R \text {. }
$$

Using Strong Collection we get that

$$
\left(\forall X \in A^{\prime}\right)\left(\exists Y \in A^{\prime}\right)(X, Y) \in R^{\prime} .
$$

As $a \in A^{\prime}$ we may apply $\mathbf{R D C}$ to get a function $f: \mathbb{N} \rightarrow A^{\prime}$ such that $f(0)=$ $a$ and $(f(n), f(n+1)) \in R^{\prime}$ for all $n \in \mathbb{N}$. Now let $b=\bigcup_{n \in \mathbb{N}} f(n)$. Then $a \subseteq b$. Also if $x \in b$ then $x \in f(n)$ for some $n \in \mathbb{N}$ and, as $(f(n), f(n+1)) \in R^{\prime}$, there is $y \in f(n+1) \subseteq b$ such that $(x, y) \in R$. Thus $(\forall x \in b)(\exists y \in b)(x, y) \in R$.

Finally we prove each instance of RDC using RRS and DC. So let $A, R$ be classes with $R \subseteq A \times A$, such that $(\forall x \in A)(\exists y \in A)(x, y) \in R$, and let $a_{0} \in A$. Then, by RRS, there is a set $b \subseteq A$ such that $\left\{a_{0}\right\} \subseteq b$ and

$$
(\forall x \in b)(\exists y \in b)(x, y) \in R .
$$

By Strong Collection there is a set $r \subseteq R$ such that $(\forall x \in b)(\exists y \in b)(x, y) \in$ $r$. So, by DC, there is $f: \mathbb{N} \rightarrow b$ such that $f(0)=a_{0}$ and, for all $n \in \mathbb{N}$, $(f(n), f(n+1)) \in r \subseteq R$.

We end this section with a proof of the result that the new scheme is a theorem of classical set theory, the proof using a standard technique of classical set theory for proving reflection properties. This is to use the cumulative hierarchy $\left\{V_{\alpha}\right\}_{\alpha \in O n}$ of sets $V_{\alpha}$ indexed by ordinals $\alpha \in O n$. This hierarchy is given by the recursive defining equation

$$
V_{\alpha}=\bigcup_{\beta<\alpha} \operatorname{Pow}\left(V_{\beta}\right)
$$

and has the property that $V_{\beta} \subseteq V_{\alpha}$ for $\beta<\alpha$. Moreover if $V$ is the universe class of all sets then $V=\bigcup_{\alpha \in O n} V_{\alpha}$.

Theorem: 2.5 Each instance of $\mathbf{R R S}$ is a theorem of $\mathbf{Z F}$. 


\section{Proof:}

Let $A, R$ be classes with $R \subseteq A \times A$, such that $(\forall x \in A)(\exists y \in A)(x, y) \in$ $R$, and let $a$ be a subset of $A$. We must show that there is a subset $b$ of $A$ such that $a \subseteq b$ and

$$
(\forall x \in b)(\exists y \in b)(x, y) \in R .
$$

For each ordinal $\alpha \in O n$ let $A_{\alpha}=A \cap V_{\alpha}$. Then each $A_{\alpha}$ is a set, $A_{\beta} \subseteq A_{\alpha}$ for $\beta<\alpha$ and $A=\bigcup_{\alpha \in O n} A_{\alpha}$.

Let $\alpha \in O n$. If $x \in A_{\alpha}$ then there is $\beta \in O n$ such that $\left(\exists y \in A_{\beta}\right)[(x, y) \in$ $R]$ annd so we may let $f_{\alpha}(x)$ be the least such $\beta$. Let $g(\alpha)=\sup _{x \in A_{\alpha}} f_{\alpha}(x)$. Then $\left(\forall x \in A_{\alpha}\right)\left(\exists y \in A_{g(\alpha)}\right)[(x, y) \in R]$. As the set $a$ is a subset of $A$ it is a subset of $A_{\alpha_{0}}$ for some ordinal $\alpha_{0}$. By primitive recursion we can define a strictly increasing infinite sequence of ordinals $\alpha_{0}<\alpha_{1}<\cdots$ by letting $\alpha_{n+1}=\max \left(g\left(\alpha_{n}\right), \alpha_{n}+1\right)$ for each $n \in \mathbb{N}$. Let $\alpha=\sup _{n \in \mathbb{N}} \alpha_{n}$. Then

$$
\begin{aligned}
x \in A_{\alpha} & \Rightarrow x \in A_{\alpha_{n}} & \text { for some } n \in \mathbb{N} \\
& \Rightarrow(x, y) \in R & \text { for some } y \in A_{\alpha_{n+1}} \\
& \Rightarrow(x, y) \in R & \text { for some } y \in A_{\alpha}
\end{aligned}
$$

Now if $b=A_{\alpha}$ then $a \subseteq A_{\alpha_{0}} \subseteq A_{\alpha}$ and $(\forall x \in b)(\exists y \in b)(x, y) \in R$, as desired.

\section{Coinductive definitions of classes}

It has turned out there there are results which have been proved using RDC, but in fact only need RRS. In particular we have a result concerning the coinductive definition of classes in constructive set theory which is a dual to a result concerning the inductive definition of classes. While the inductive definitions result can be carried out in $\mathbf{C Z F}^{-}$the dual result has seemed to need RDC, but in fact can be proved using just RRS.

Recall from [4] that given a class $\Phi$ of pairs $(X, a)$ it can be proved in $\mathbf{C Z F}^{-}$that there is a smallest $\Phi$-closed class $I$, where a class $Y$ is $\Phi$-closed if, for every $(X, a) \in \Phi$,

$$
X \subseteq Y \Rightarrow a \in Y
$$

We call $I$ the class inductively defined by $\Phi$.

As with inductive definitions a coinductive definition can also be given by a class $\Phi$ of pairs $(X, a)$. A class $Y$ is defined to be $\Phi$-progressive if, for 
every $(X, a) \in \Phi$,

$$
a \in Y \Rightarrow X \backslash Y \text {, }
$$

where $X \backslash Y$ if $X \cap Y$ is inhabited; i.e. has an element. If there is a largest $\Phi$-progressive class $J$ then we call $J$ the class coinductively defined by $\Phi$.

Note that, using classical logic, a class $Y$ is $\Phi$-progressive iff its complement class $\{a \mid a \notin Y\}$ is $\Phi$-closed and hence when the smallest $\Phi$-closed class $I$ exists then its complement is the largest $\Phi$-progressive class $J$. So, in $\mathbf{Z F}, J$ always exists. The following result seems to be the best we can do in constructive set theory.

Theorem: $3.1\left(\mathbf{C Z F}^{-}+\mathbf{R R S}\right)$ Let $\Phi$ be a class of pairs $(X, a)$ such that the class $\Phi_{a}=\{X \mid(X, a) \in \Phi\}$ is a set for all sets a. Then there is a largest $\Phi$-progressive class $J$.

First observe that the union of any family of $\Phi$-progressive classes is $\Phi$ progressive. In particular the class

$$
J=\bigcup\{Y \mid Y \text { is a } \Phi \text {-progressive set }\}
$$

is a $\Phi$-progressive class. We will show that any $\Phi$-progressive class $B$ is a subclass of $J$. We will use the following lemma.

Lemma 3.2 If $B$ is a $\Phi$-progressive class then $\forall Y \in P$ Pow $(B) \exists Y^{\prime} \in \operatorname{Pow}(B) \forall a \in$ $Y \forall X \in \Phi_{a} X \backslash Y^{\prime}$.

Proof: Given $Y \in \operatorname{Pow}(B)$ let $\Phi^{Y}=\{(X, a) \in \Phi \mid a \in Y\}$. Then $\Phi^{Y}=\bigcup_{a \in Y}\left(\Phi_{a} \times\{a\}\right)$ is a set and, as $B$ is $\Phi$-progressive and $Y \subseteq B$,

$$
\left(\forall(X, a) \in \Phi^{Y}\right) \exists y[y \in X \cap B] .
$$

So, by Strong Collection, there is a set $Y^{\prime}$ such that

$$
\left(\forall(X, a) \in \Phi^{Y}\right)\left(\exists y \in Y^{\prime}\right)[y \in X \cap B] \&\left(\forall y \in Y^{\prime}\right)\left(\exists(X, a) \in \Phi^{Y}\right)[y \in X \cap B] .
$$

So $Y^{\prime} \in \operatorname{Pow}(B)$ and $a \in Y \Rightarrow X \backslash Y^{\prime}$, for all $(X, a) \in \Phi$, completing the proof of the lemma. 
Proof of Theorem 3.1: $\quad$ To show that a $\Phi$-progressive class $B$ is a subclass of $J$ let $b \in B$. It suffices to show that $b \in U$ for some $\Phi$-progressive set $U$, as then $b \in U \subseteq J$. By the lemma and RRS there is a set $Z \subseteq \operatorname{Pow}(B)$ such that $\{b\} \in Z$ and

$$
\text { (*) } \forall Y \in Z \exists Y^{\prime} \in Z \forall a \in Y \forall X \in \Phi_{a} X \backslash Y^{\prime} .
$$

Note that, using RDC instead of RRS, a set $Z$ such that $\{b\} \in Z$ and (*) can be defined to be $\bigcup_{n \in \mathbb{N}} f(n)$ where $f: \mathbb{N} \rightarrow$ Pow $(B)$ is obtained using the lemma and RDC, so that $f(0)=\{b\}$ and, for all $n \in \mathbb{N}$,

$$
\left.\forall a \in f(n) \forall X \in \Phi_{a} X\right\rceil f(n+1) .
$$

Let $U=\cup Z$. Then $b \in U$. Also if $a \in U$, with $(X, a) \in \Phi$, then $a \in Y$ for some $Y \in Z$ so that $X \backslash Y^{\prime}$ for some $Y^{\prime} \in Z$ and hence $X \backslash U$. Thus $U$ is a $\Phi$-progressive set.

We have now shown that $J$ is the largest $\Phi$-progressive class.

\section{Review of the Heyting algebra valued Models of $\mathrm{CZF}^{-}$}

While $\mathbf{R D C}$ is obviously a choice principle it would seem that RRS may not be a choice principle. One evidence for this is the following fact. The choice principles RDC and DC are not generally preserved in the $\mathrm{cHa}$ models for $\mathbf{C Z F}^{-}$but the scheme RRS is; i.e. we have the following result.

Theorem: 4.1 ( $\left.\mathbf{C Z F}^{-}+\mathbf{R R S}\right)$ The scheme $\mathbf{R R S}$ holds in each cHa model of $\mathrm{CZF}^{-}$.

We will sketch a proof of this theorem. But first we review the $\mathrm{cHa}$ model construction.

Nicola Gambino, in his $\mathrm{PhD}$ thesis, [5], and subsequently in [6], has shown how to carry over the apparatus of Boolean valued models of the classical set theory $Z F$ in the classical metatheory of $Z F$ that uses complete Boolean algebras, to the more general context of Heyting algebra valued models of $\mathbf{C Z F}^{-}$in the constructive metatheory of $\mathbf{C Z F}^{-}$. For this it is necessary to use a $\mathrm{cHa}$ (complete Heyting algebra) that is set generated. Moreover Gambino assumes, without loss of generality, that the set-generated $\mathrm{cHa}$ is given in terms of a poset $S$; i.e. a set that comes equipped with a partial 
ordering that is itself a set of ordered pairs. The elements of the $\mathrm{cHa}$ are the $j$-closed subsets of a poset $S$, where $j: \operatorname{Pow}(S) \rightarrow \operatorname{Pow}(S)$ satisfies the following conditions for $a, b \in \operatorname{Pow}(S)$ and $a \in \operatorname{Pow}(S)$ is $j$-closed if $j a \subseteq a$. We call $a$ a lower set if

$$
s \leq s^{\prime} \in a \Rightarrow s \in a
$$

1. $j a$ is a lower set,

2. $a \subseteq j a$,

3. $j(j a) \subseteq j a$,

4. $a \subseteq b \Rightarrow j a \subseteq j b$,

5. $j a \cap j b \subseteq j(a \cap b)$ if $a, b$ are lower sets.

We will call an operator $j$ satisfying the conditions $1-5$ above a local closure operator.

The class of lower sets of the poset $S$ form a set-based topology on $S$ and so form a set-generated $c H a$, partially ordered by the subset relation, and the local closure operator $j$, when restricted to the lower sets, is a nucleus. It follows that the class $\Omega_{j} S$ of $j$-closed sets also form a set-generated $\mathrm{cHa}$, when partially ordered by the subset relation. Every set-generated $\mathrm{cHa}$ is isomorphic to one of the form $\Omega_{j} S$. So there is no real loss in just considering such set-generated $\mathrm{cHa}$ 's. The advantage in working with a $\mathrm{cHa}$ of the form $\Omega_{j} S$ is that there is a good notion of $j$-closed subclass of $S$. A class $P \subseteq S$ is defined to be a $j$-closed class if $j a \subseteq P$ for every set $a \subseteq P$. For each class $P \subseteq S$ let $J P=\bigcup\{j a \mid a \subseteq P\}$. So $P$ is $j$-closed iff $J P \subseteq P$. The indexed sup and inf operations on the $j$-closed classes is defined as follows, where $P_{i}$ is a $j$-closed class for each $i \in L$, with $I$ a class.

$$
\begin{aligned}
& \bigvee_{i \in I} P_{i}=J\left(\bigcup_{i \in I} P_{i}\right) \\
& \bigwedge_{i \in I} P_{i}=\bigcap_{i \in I}^{\prime} P_{i}=\left\{s \in S \mid(\forall i \in I) s \in P_{i}\right\}
\end{aligned}
$$

Both $\bigvee_{i \in I} P_{i}$ and $\bigwedge_{i \in I} P_{i}$ are $j$-closed classes and are sets when $I$ and each $P_{i}$ are sets. We get the binary join and meet operations on the $j$-closed classes by taking $I=2$ to get $P_{0} \vee P_{1}=\bigvee_{i \in 2} P_{i}$ and $P_{0} \wedge P_{1}=\bigwedge_{i \in 2} P_{i}$. We also need the pseudo-complement operation on the $j$-closed classes. For $j$-closed classes $P, Q$ we let

$$
P \rightarrow Q=\left\{s \in S \mid\left(\forall s^{\prime} \leq s\right)\left[s^{\prime} \in P \Rightarrow s^{\prime} \in Q\right]\right\} .
$$


Then $P \rightarrow Q$ is also $j$-closed and is a set if $P, Q$ are sets.

We are now ready to define the model. From now on we will just write $\Omega^{\prime}$ for $\Omega_{j} S$. The universe of the model is inductively defined to be the smallest class $V^{\prime}$ such that

if $a$ is a function $a: \delta a \rightarrow \Omega^{\prime}$ with domain $\delta a$ a subset of $V^{\prime}$ then $a \in V^{\prime}$.

If $a \in V^{\prime}$ and $P_{b}$ is a $j$-closed class for each $b \in \delta a$ then let

$$
\begin{aligned}
& \bigvee_{b: a} P_{b}=\bigvee_{b \in \delta a}\left[a(b) \wedge P_{b}\right] \\
& \bigwedge_{b: a} P_{b}=\bigwedge_{b \in \delta a}\left[a(b) \rightarrow P_{b}\right]
\end{aligned}
$$

We define $E q(a, b) \in V^{\prime}$ for $a, b \in V^{\prime}$ by a double recursion on the inductive definition of $V^{\prime}$ using the following equation.

$$
E q(a, b)=\bigwedge \bigvee_{a^{\prime}: a} E q\left(a^{\prime}, b^{\prime}\right) \wedge \bigwedge_{b^{\prime}: b} \bigvee_{a^{\prime}: a} E q\left(a^{\prime}, b^{\prime}\right)
$$

Let $\mathcal{L}$ be the following first order language of set theory. As well as the logical constants $T, \perp$, binary connectives $\wedge, \vee, \rightarrow$, quantified variables $\forall x, \exists x$ and equality predicate symbol $=$ the language has primitive restricted quantifiers $(\forall x \in y),(\exists x \in y)$. For any class $A$ let $\mathcal{L} A$ be the language obtained from $\mathcal{L}$ by adding an individual constant for each element of $A$. It will be convenient to identify the element with its constant. So the terms of $\mathcal{L} A$ are either the variables of $\mathcal{L}$ or the constants from $A$ and the formulae of $\mathcal{L} A$ are generated using the following rules.

1. Every atomic formula $(s=t)$ is a formula where $s, t$ are terms of $\mathcal{L} A$,

2. $\top, \perp$ are formulae.

3. If $\phi, \psi$ are formulae then so are $\phi \wedge \psi, \phi \vee \psi$ and $\phi \rightarrow \psi$.

4. If $\phi$ is a formula so are $(\forall x \in y) \phi,(\exists x \in y)$.

5. If $\phi$ is a formula so are $\forall x \phi, \exists x \phi$.

The $\Delta_{0}$ formulae are those generated using only rules $1-4$. We use the usual definitions for $\neg \phi$ and $(\phi \leftrightarrow \psi)$ as $(\phi \rightarrow \perp)$ and $(\phi \rightarrow \psi) \wedge(\psi \rightarrow \phi)$ respectively. In addition we use the definition

$$
(x \in y) \equiv(\exists z \in y)[x=z] .
$$


We now associate a $j$-closed class $[[\phi]]_{V^{\prime}}$ to each sentence $\phi$ of $\mathcal{L} V^{\prime}$ by recursion following the way the sentence $\phi$ is generated. In these equations we have left off the subscript $V^{\prime}$ from $[[]]_{V^{\prime}}$ and added a subscript $\Omega^{\prime}$ to the elements $\top, \perp$ of $\Omega^{\prime}$ and the three binary $H a$ operations $\wedge, \vee, \rightarrow$ on the $j$ closed classes so as to avoid any possible confusion with the logical constants and binary connectives.

1. $[[a=b]]=E q(a, b)$ for $a, b \in V^{\prime}$,

2. $[[\top]]=\top_{\Omega^{\prime}}$ and $[[\perp]]=\perp_{\Omega^{\prime}}$,

3. $[[\phi \wedge \psi]]=[[\phi]] \wedge_{\Omega^{\prime}}[[\psi]], \quad[[\phi \vee \psi]]=[[\phi]] \vee_{\Omega^{\prime}}[[\psi]]$ and $[[\phi \rightarrow \psi]]=$ $[[\phi]] \rightarrow \Omega^{\prime}[[\psi]]$

4. $[[(\forall x \in a) \phi(x)]]=\bigwedge_{a^{\prime}: a}\left[\left[\phi\left(a^{\prime}\right)\right]\right]$ and $[[(\exists x \in a) \phi(x)]]=\bigvee_{a^{\prime}: a}\left[\left[\phi\left(a^{\prime}\right)\right]\right]$,

5. $[[\forall x \phi(x)]]=\bigwedge_{a^{\prime} \in V^{\prime}}\left[\left[\phi\left(a^{\prime}\right)\right]\right]$, and $[[\exists x \phi(x)]]=\bigvee_{a^{\prime} \in V^{\prime}}\left[\left[\phi\left(a^{\prime}\right)\right]\right]$.

Note that $[[\phi]]$ is a set and so in $\Omega^{\prime}$ when $\phi$ is a $\Delta_{0}$ sentence of $\mathcal{L} V^{\prime}$.

A sentence $\phi$ of $\mathcal{L} V^{\prime}$ is valid in $V^{\prime}$, written $\models_{V^{\prime}} \phi$, if $[[\phi]]_{V^{\prime}}=T_{\Omega^{\prime}}$. A formula $\phi\left(x_{1}, \ldots, x_{n}\right)$ of $\mathcal{L} V^{\prime}$ is valid in $V^{\prime}$, written $\models_{V^{\prime}} \phi\left(x_{1}, \ldots, x_{n}\right)$, if its universal closure $\forall x_{1} \cdots \forall x_{n} \phi\left(x_{1}, \ldots, x_{n}\right)$ is valid in $V^{\prime}$; i.e. $\models_{V^{\prime}} \phi\left(a_{1}, \ldots, a_{n}\right)$ for all $a_{1}, \ldots, a_{n} \in V^{\prime}$.

The following result is essentially Theorem 3.15 of [6].

Theorem: 4.2 Assuming $\mathbf{C Z F}^{-}$in the metatheory, each theorem of $\mathbf{C Z F}^{-}$ is valid in $V^{\prime}$. Moreover, if $\mathbf{C Z F}$ is assumed in the metatheory and $\Omega^{\prime}$ is set-presented then each theorem of $\mathbf{C Z F}$ is valid in $V^{\prime}$.

\section{$5 \quad$ The proof of Theorem 4.1}

We want to show, in $\mathbf{C Z F}{ }^{-}+\mathbf{R R S}$, that every instance of the scheme $\mathbf{R R S}$ is valid in the $c H a$ semantics over $\Omega^{\prime}$. So let $\theta(x)$ and $\phi(x, y)$ be formulae of $\mathcal{L} V^{\prime}$ having at most the free variables displayed. We want to prove the validity in $V^{\prime}$ of the sentence

$$
\forall u\left[\phi^{\prime} \wedge \theta^{\prime}(u) \rightarrow \exists v\left(u \subseteq v \wedge \theta^{\prime}(v) \wedge(\forall x \in v)(\exists y \in v) \phi(x, y)\right)\right],
$$

where $\phi^{\prime} \equiv \forall x[\theta(x) \rightarrow \exists y(\theta(y) \wedge \phi(x, y))]$ and $\theta^{\prime}(u) \equiv \forall x \in u \theta(x)$. To do that it is sufficient to prove the following result.

Theorem: 5.1 Let $p \in \Omega^{\prime}$ and $a_{0} \in V^{\prime}$ such that

$1 p \subseteq[[\forall x[\theta(x) \rightarrow \exists y(\theta(y) \wedge \phi(x, y))]]]$, 
$2 p \subseteq\left[\left[\forall x \in a_{0} \theta(x)\right]\right]$.

Then there is $b \in V^{\prime}$ such that

$3 p \subseteq\left[\left[a_{0} \subseteq b\right]\right]$,

$4 p \subseteq[[\forall x \in b \theta(x)]]$,

$5 p \subseteq[[(\forall x \in b)(\exists y \in b) \phi(x, y)]]$.

Proof: Let $p \in \Omega^{\prime}$ such that 1 and let $A=\left\{a \in V^{\prime} \mid p \subseteq[[\forall x \in a \theta(x)]]\right\}$.

Claim: $\quad(\forall a \in A)(\exists b \in A) \quad p \subseteq[[(\forall x \in a)(\exists y \in b) \phi(x, y)]]$.

Proof of Claim: Let $a \in A$. Then, by $\mathbf{1}, p \subseteq[[(\forall x \in$ a) $(\exists y)(\theta(y) \wedge \phi(x, y))]]$. As in the proof of Proposition 5.17 of [5] or Proposition 3.11 of [6] we get $b \in V^{\prime}$ such that $p \subseteq$ $[[(\forall x \in a)(\exists y \in b)(\theta(y) \wedge \phi(x, y))]]$ and $p \subseteq[[(\forall y \in b)(\exists x \in$ a) $(\theta(y) \wedge \phi(x, y))]]$. It follows that $b \in A$ and $p \subseteq[[(\forall x \in a)(\exists y \in$ b) $\phi(x, y)]]$.

Now let $a_{0} \in V^{\prime}$ such that 2; i.e. $a_{0} \in A$. Let $\bar{a}=\left\{a_{0}\right\}$. So $\bar{a} \subseteq A$ and, using the claim, we may apply $\mathbf{R R S}$ to get a set $\bar{b} \subseteq A$ such that $\bar{a} \subseteq \bar{b}$ and

$(*) \quad(\forall a \in \bar{b})\left(\exists a^{\prime} \in \bar{b}\right) p \subseteq\left[\left[(\forall x \in a)\left(\exists y \in a^{\prime}\right) \phi(x, y)\right]\right]$.

Define $b \in V^{\prime}$ as follows. Let $\delta b=\bigcup\{\delta a \mid a \in \bar{b}\}$. Then $\delta b$ is a subset of $V^{\prime}$ and we can define $b: \delta b \rightarrow \Omega^{\prime}$ as follow. For $c \in \delta b$ let $b(c)=\bigvee_{a \in \bar{b}_{c}} a(c)$, where $\bar{b}_{c}=\{a \in \bar{b} \mid c \in \delta a\}$. It only remains to prove $\mathbf{3}, \mathbf{4}, \mathbf{5}$.

Proof of 3 Note that $a_{0} \in \bar{b}$ and $\left[\left[a_{0} \subseteq b\right]\right]=\bigwedge_{c \in \delta a_{0}}\left(a_{0}(c) \rightarrow \bigvee_{c^{\prime} \in \delta b}\left(b\left(c^{\prime}\right) \wedge\right.\right.$ $\left.\left.\left[\left[c=c^{\prime}\right]\right]\right)\right)$. But, for $c \in \delta a_{0}$, if $c^{\prime}=c$ then $c^{\prime} \in \delta b$ and, as $a_{0} \in \bar{b}_{c}$ and $\left[\left[c=c^{\prime}\right]\right]=\top$,

$$
\left.a_{0}(c) \subseteq\left(b\left(c^{\prime}\right) \wedge\left[\left[c=c^{\prime}\right]\right]\right) \subseteq \bigvee_{c^{\prime} \in \delta b}\left(b\left(c^{\prime}\right) \wedge\left[\left[c=c^{\prime}\right]\right]\right)\right)
$$

So $p \subseteq \top=\left[\left[a_{0} \subseteq b\right]\right]$ and, in fact, $\models a_{0} \subseteq b$. 
Proof of 4 Observe that

$$
\begin{aligned}
{[[\forall x \in b \theta(x)]] } & =\bigwedge_{c \in \delta b}(b(c) \rightarrow[[\theta(c)]]) \\
& =\bigwedge_{c \in \delta b}\left(\bigvee_{a \in \bar{b}_{c}} a(c) \rightarrow[[\theta(c)]]\right) \\
& =\bigwedge_{c \in \delta b} \bigwedge_{a \in \bar{b}_{c}}(a(c) \rightarrow[[\theta(c)]]) \\
& =\bigwedge_{a \in \bar{b}} \bigwedge_{c \in \delta a}(a(c) \rightarrow[[\theta(c)]]) \\
& =\bigwedge_{a \in \bar{b}}[[\forall x \in a \theta(x)]]
\end{aligned}
$$

But, as $\bar{b} \subseteq A, p \subseteq[[\forall x \in a \theta(x)]]$ for all $a \in \bar{b}$ so that $p \subseteq[[\forall x \in$ $b \theta(x)]]$.

Proof of 5 It suffices to show that, for $c \in \delta b,(p \wedge b(c)) \subseteq \bigvee_{c^{\prime} \in \delta b}\left(b\left(c^{\prime}\right) \wedge\right.$ $\left.\left[\left[\phi\left(c, c^{\prime}\right)\right]\right]\right)$. As $b(c)=\bigvee_{a \in \bar{b}_{c}} a(c)$ it suffices to show that, for each $a \in \bar{b}_{c}$,

$$
p \wedge a(c) \subseteq \bigvee_{c^{\prime} \in \delta b}\left(b\left(c^{\prime}\right) \wedge\left[\left[\phi\left(c, c^{\prime}\right)\right]\right]\right) .
$$

Now if $a \in \bar{b}_{c}$ then $a \in \bar{b}$ so that, by $(*)$, there is $a^{\prime} \in \bar{b}$ such that

$$
p \subseteq\left[\left[(\forall x \in a)\left(\exists y \in a^{\prime}\right) \phi(x, y)\right]\right] .
$$

It follows that, as $c \in \delta a$,

$$
\begin{aligned}
p \wedge a(c) & \subseteq\left[\left[\exists y \in a^{\prime} \phi(c, y)\right]\right] \\
& =\bigvee_{c^{\prime} \in \delta a^{\prime}}\left(a^{\prime}\left(c^{\prime}\right) \wedge\left[\left[\phi\left(c, c^{\prime}\right)\right]\right]\right) \\
& \subseteq \bigvee_{c^{\prime} \in \delta b}\left(b\left(c^{\prime}\right) \wedge\left[\left[\phi\left(c, c^{\prime}\right)\right]\right]\right),
\end{aligned}
$$

as was wanted.

\section{References}

[1] P. Aczel. The type theoretic interpretation of constructive set theory. In MacIntyre, A. and Pacholski, L. and Paris, J., editors, Logic Colloquium 'r7', Amsterdam, North Holland, pp. 55-66 (1978). 
[2] P. Aczel. The type theoretic interpretation of constructive set theory: Choice principles. In S.S. Troelstra and D. van Dalen, editors, The L.E.J. Brouwer Centenary Symposium, Amsterdam, North Holland, pp. 1-40 (1982).

[3] P. Aczel. The type theoretic interpretation of constructive set theory: Inductive definitions. In R.B. et al. Marcus, editor, Logic, Methodology and Philosophy of Science VII, Amsterdam, North Holland, pp. 17-49 (1986).

[4] P. Aczel and M. Rathjen, Notes on Constructive Set Theory, Mittag-Leffler Technical Report No. 40, 2000/2001, (2001).

[5] N. Gambino, Sheaf interpretations for generalised predicative intuitionistic systems, PhD thesis, Department of Computer Science, University of Manchester, (2002).

[6] N. Gambino, Heyting-valued interpretations for Constructive Set Theory, Annals of Pure and Applied Logic, 137 (1-3), pp 164-188, (2006).

[7] P. Martin-Löf. Intuitionistic Type Theory, Studies in Proof Theory, Bibiopolis, (1984).

[8] J. Myhill. Constructive Set Theory, Journal of Symbolic Logic, 40, pp 47-382, (1975).

[9] A.S Troelstra and D. van Dalen. Constructivism in Mathematics: An Introduction (two volumes), North Holland, (1988). 